\title{
Preliminary Development of Solid Dispersion for an Insoluble Compound ZL006 by Miniaturized Hot Melt Extrusion
}

\author{
Lijun Zhang ${ }^{1,2}$ Hansen Luan ${ }^{2}$ Weiyue Lu ${ }^{1}$ Hao Wang ${ }^{2}$ \\ ${ }^{1}$ Department of Pharmaceutics, School of Pharmacy, Fudan \\ University, Shanghai, China \\ 2 Department of Microspheres Technology, National Pharmaceutical \\ Engineering Research Center, China State Institute of

\begin{abstract}
Address for correspondence Weiyue Lu, Department of Pharmaceutics, School of Pharmacy, Fudan University, 826 Zhangheng Road, Shanghai, 201203, China

(e-mail: wylu@shmu.edu.cn; wanghao99@hotmail.com).
\end{abstract}

Pharmaceutical Industry, Shanghai, China

Pharmaceut Fronts 2019;1:e11-e21.

\begin{abstract}
Keywords

- solid dispersion

- hot-melt extrusion

- miniaturized

- Soluplus

- hydroxypropyl methylcellulose acetate succinate

- ZL006

The purpose of this study was to develop a solid dispersion (SD) by miniaturized hot-melt extrusion (HME) for an insoluble molecule ZL006 which showed potency of increasing leukocytes. A preliminary formulation screening was conducted using solvent evaporation method. The selected SD formulation was further optimized and scaled up using a miniaturized twin-screw extruder. Solid-state characterizations of the scale-up SD and its corresponding physical mixture (PM) were performed by X-ray powder diffraction (XRPD), modulated differential scanning calorimetry (mDSC), and Fourier's transform infrared spectroscopy (FTIR). XRPD and mDSC results indicated the formation of amorphous SD. FTIR spectrum indicated the possible hydrogen bond formation between the compound and the excipient. A discriminating non-sink condition micro-dissolution of SD showed the fast release of ZL006 which was approximately two-fold and three-fold of dissolution of PM and pure crystalline compound, respectively. The preliminary in vivo pharmacokinetics (PK) study in rats showed $71 \%$ oral bioavailability from the SD, while the bioavailability of ZL006 conventional suspension was less than 1\%. Thus, an SD formulation for ZL006 with improved solubility and bioavailability was developed by miniaturized HME with minimal amount of compound at early preclinical stage, which could enable the preclinical evaluation.
\end{abstract}

\section{Introduction}

ZL006 (-Fig. 1) is a novel molecule at preclinical stage, which was discovered to be able to promote peripheral leukocytes after leukopenia induced by chemotherapeutic agents like 5-fluorouracil in animal models by Shanghai Institute of Pharmaceutical Industry (SIPI). Pharmacological studies indicated that ZL006 could induce the hemopoietic stem cells of human and mice to differentiate, which was similar to the mode of action of human granulocyte colony stimulating factor (G-CSF). ${ }^{1}$ Thus, it was investigated as a potential drug candidate for the development of orally administered small molecule to promote leukocytes proliferation, and to avoid the potential risks of manufacturing, storage, and shipment of biologics and also to reduce the cost of use. However, because of its extremely poor solubility and moderate permeability, so far there have been no improved physical forms (salts or cocrystals) and no oral bioavailability enhancing formulations to provide enough in vivo exposure for its preliminary toxicity evaluation which has been the hurdle for preclinical studies.

It is well known that inadequate exposure in efficacy and toxicity studies due to poor solubility and low bioavailability can lead to misleading evaluation for the compound of interest. ${ }^{2}$ Therefore, several strategies to enhance oral bioavailability of poorly water-soluble compounds during the drug development process have been applied and reported. ${ }^{3,4}$ Solid dispersion (SD) prepared by hot-melt extrusion (HME) is one of the most commonly used strategies which has been successfully applied in many studies. ${ }^{4-10}$ It is already common understanding that not only the 


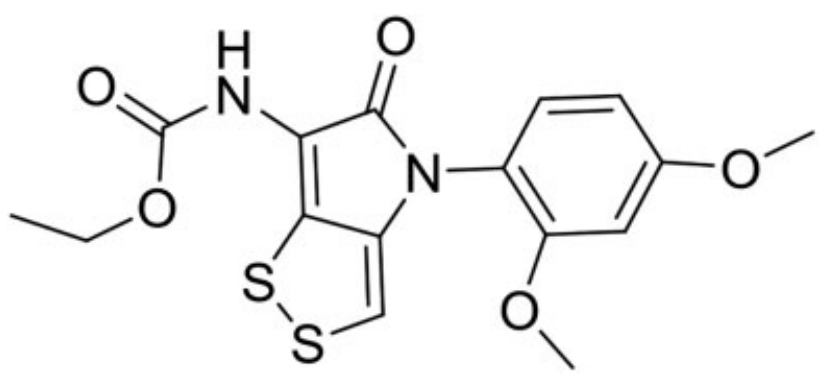

Fig. 1 Chemical structure of ZL006.

formulation composition but also the process parameters and screw configurations have significant impacts on the product attributes, including dissolution rate, bioavailability, and stability. ${ }^{8,10-15}$ However, the availability of compound at early development or even research stage is usually limited. Thus, miniaturized formulation screening method and hotmelt extruder are required to fit the purpose for SD development at early development stage and save the consumption of the compound. Though the extruder is miniscale, some basic process parameters, such as extrusion temperature and screw speed, are still required to be investigated. Currently, only few papers discussed the systematic development of SD using miniscale or miniaturized HME with limited amount of active pharmaceutical ingredient (API) at early development stage. ${ }^{9,16,17}$

The objective for this paper is to improve oral bioavailability of ZL006 by developing a solid dispersion via miniaturized HME with minimal material resources.

\section{Materials and Methods}

\section{Materials}

ZL006 was provided by SIPI with purity $>99 \%$. Soluplus, polyvinylpyrrolidone (PVP) K30, PVP K90, PVP-VA64, Cremophor RH40, Solutol HS15, poloxamer 188, and poloxamer 407 were all obtained from BASF chemical company (Ludwigshafen, Germany). Hydroxypropyl methylcellulose-acetate succinate-LF (HPMCAS-LF) and hydroxypropyl methylcellulose phthalate (HPMC-P) were purchased from Shin-Etsu Chemical Co., Ltd (Tokyo, Japan). HPMC-E3 was obtained from Dow Chemicals. Hydroxypropyl cellulose (HPC-EF and HPC-L) were obtained from NISSO (Tokyo, Japan). Methacrylic acid copolymers (Eudragit EPO and Eudragit L100) were provided by Evonik Rohm GmbH (Darmstadt, Germany). $D$ - $\alpha$-tocopheryl polyethylene glycol 1,000 succinate (Vitamin E TPGS) was obtained from Eastman Chemical Company. Sodium lauryl sulfate (SLS), PEG (polyethylene glycol)-8000, carboxy methyl cellulose sodium (CMC-Na), and polysorbate 80 (Tween 80 ) were purchased from Sigma-Aldrich (St. Louis, MO, USA). All reagents and chemicals were analytical grade and used as received. Male SD rats weighing $200 \pm 20$ g were purchased from Shanghai, SIPPR-BK Laboratory Animal Co., Ltd., with qualification certificate number 2008001665579.

\section{SD Formulation Screening by Solvent Evaporation}

Polymer Screening

Different polymers stock solutions were prepared in 1:1 mixture of dichloromethane-ethanol at $50 \mathrm{mg} / \mathrm{mL}$ and ZL006 was dissolved in dichloromethane at $20 \mathrm{mg} / \mathrm{mL}$ as compound stock solution. Dispense $400 \mu \mathrm{L}$ of each polymer stock solution and $250 \mu \mathrm{L}$ of ZL006 stock solution to a glass vial, mix, and vortex. Then the solvents were removed in a vacuum oven at $40^{\circ} \mathrm{C}$, and the obtained solid dispersions with $20 \%$ drug load were further dried under vacuum at $40^{\circ} \mathrm{C}$ overnight. Miniaturized two-step kinetic solubility of the solid dispersions and X-ray powder diffraction (XRPD) were tested.

\section{Surfactant Screening}

Solubility test of ZL006 in different surfactant water solutions was conducted to select the optimal surfactant. Briefly, after preparing the surfactant solutions in water at 5 or $10 \%$ $(\mathrm{w} / \mathrm{v})$ concentration, the excess amounts of ZL006 were added to the above surfactant solutions, followed by stirring the samples at room temperature for 18 to 24 hours to reach the equilibrium solubility. Filtrate the samples with 96-well filter plate $(0.45 \mu \mathrm{m}$ polyvinylidene fluoride [PVDF] membrane, Millipore) by centrifugation at $4,000 \mathrm{r} / \mathrm{min}$ for 5 minutes, dilute the filtered solutions with the diluent (the mixture of $80 \%$ acetonitrile and $20 \%$ water), and inject $1 \mu \mathrm{L}$ to a UPLC system, which is described in section, "Miniaturized Two-Step Kinetic Solubility Test."

\section{Solid Dispersion Formulation Screening}

Based on the polymer screening and surfactant screening results, different SD formulation variants were prepared by solvent evaporation. Briefly, the required excipients stock solutions and ZL006 stock solution were dispensed in a glass vial and the rest procedures were the same as the above mentioned in section, "Polymer Screening." Miniaturized two-step kinetic solubilities of different SD formulations were determined to select the lead SD formulation.

\section{Optimization and Scale up of SD Formulation by HME Process}

\section{Orthogonal Design of Experiments}

Based on the lead SD formulation, an orthogonal design of experiments including three factors and three levels was applied to optimize the formulation and HME process parameters. Nine formulation variants were listed in - Table 1, including drug load from 10 to $20 \%$, process temperature from 160 to $180^{\circ} \mathrm{C}$ and screw speed from 100 to $200 \mathrm{rpm}$. For each variant, $600 \mathrm{mg}$ of required excipients and ZL006 were mixed by mortar and pestle, and the powder blend was then manually fed into a miniscale extruder (ZE5, ThreeTec, Switzerland) which was equipped with corotating twin screws of $5 \mathrm{~mm}$ diameter and heated barrel with two heating zones. The extrudates exiting through a $0.75-\mathrm{mm}$ round-die opening were cooled to room temperature and collected on the aluminum foil. All the extrudates were cut into small pieces and grounded to powder by the miller 
Table 1 Orthogonal design of experiments by HME, $\mathrm{L}_{9}\left(3^{4}\right)$

\begin{tabular}{|c|c|c|c|}
\hline \multirow[t]{2}{*}{ Levels } & \multicolumn{3}{|l|}{ Factors } \\
\hline & Drug Load /\% & $\begin{array}{l}\text { Process } \\
\text { temperature } /{ }^{\circ} \mathrm{C}\end{array}$ & Screw speed/rpm \\
\hline 1 & 10 & 160 & 100 \\
\hline 2 & 15 & 170 & 150 \\
\hline 3 & 20 & 180 & 200 \\
\hline \multicolumn{4}{|c|}{ Formulations and process parameters } \\
\hline No. & Formulation composition & $\begin{array}{l}\text { Process } \\
\text { temperature } /{ }^{\circ} \mathrm{C}\end{array}$ & Screw speed/rpm \\
\hline 1 & 10\% ZL006, 40\% Soluplus, 40\% HPMCAS-LF, 10\% Vit-E-TPGS & 160 & 100 \\
\hline 2 & 10\% ZL006, 40\% Soluplus, 40\% HPMCAS-LF, 10\% Vit-E-TPGS & 170 & 150 \\
\hline 3 & 10\% ZL006, 40\% Soluplus, 40\% HPMCAS-LF, 10\% Vit-E-TPGS & 180 & 200 \\
\hline 4 & 15\% ZL006, 37.5\% Soluplus, 37.5\% HPMCAS-LF, 10\% Vit-E-TPGS & 160 & 150 \\
\hline 5 & 15\% ZL006, 37.5\% Soluplus, 37.5\% HPMCAS-LF, 10\% Vit E TPGS & 170 & 200 \\
\hline 6 & 15\% ZL006, 37.5\% Soluplus, 37.5\% HPMCAS-LF, 10\% Vit-E-TPGS & 180 & 100 \\
\hline 7 & 20\% ZL006, 35\% Soluplus, 35\% HPMCAS-LF, 10\% Vit-E-TPGS & 160 & 200 \\
\hline 8 & 20\% ZL006, 35\% Soluplus, 35\% HPMCAS-LF, 10\% Vit-E-TPGS & 170 & 100 \\
\hline 9 & 20\% ZL006, 35\% Soluplus, 35\% HPMCAS-LF, 10\% Vit-E-TPGS & 180 & 150 \\
\hline
\end{tabular}

Abbreviations: HME, hot-melt extrusion; HPMCAS-LF, hydroxypropyl methylcellulose acetate succinate-LF; Vit-E-TPGS, vitamin-E-tocopheryl polyethylene glycol 1,000 succinate.

(Tube-Mill 100 Control, IKA, Germany), followed by passing through a 35-mesh sieve. Characterization and two-step kinetic solubility were performed for the nine SD formulations powder and one-way analysis of variance (ANOVA) for the solubilities among different formulations was conducted by GraphPad Prism 6.0 software (San Diego, California, United States).

\section{Scale up of the SD}

The optimal SD formulation was scaled up at $18 \mathrm{~g}$ with 30 -fold increase compared with the screening scale by using the optimal HME process parameters.

\section{Characterization of the SD}

\section{Miniaturized Two-Step Kinetic Solubility Test}

The appropriate amounts of SD, physical mixture (PM), or the pure crystalline ZL006 samples were added into $400 \mu \mathrm{L}$ of simulated gastric fluid (SGF; equivalent to target concentration at $1 \mathrm{mg} / \mathrm{mL}$ in SGF). After stirring at room temperature for 1 hour, $200 \mu \mathrm{L}$ of the samples was withdrawn and $200 \mu \mathrm{L}$ of fasted simulated small intestinal fluid (FaSSIF) was added into the samples to keep the whole volume at $400 \mu \mathrm{L}$; then continued stirring and the samples were withdrawn at 2 and 4 hours, respectively. All the samples were filtered through a Millipore filter plate with $0.45 \mu \mathrm{m}$ PVDF membrane by centrifugation at $4,000 \mathrm{r} / \mathrm{min}$ for 5 minutes, immediately after withdrawn. The filtered samples were diluted with the diluent and $1 \mu \mathrm{L}$ of the solution was injected into a Waters Acquity UPLC system (Waters Corporation, MA, USA) with reversed phase $\mathrm{C}_{18}$ column (Acquity UPLC BEH $\mathrm{C}_{18}, 2.1 \mathrm{~mm}$ $\times 100 \mathrm{~mm}, 1.7 \mu \mathrm{m})$. The mobile phase consisted of $0.05 \%$ trifluoroacetic acid (TFA) in 95\% acetonitrile and 5\% water (A) $/ 0.05 \%$ TFA in $95 \%$ water and $5 \%$ acetonitrile (B); the gradient of $A(\%)$ was $95,5,95,95$ at $0,3,3.01$, and 4 minutes, respectively, and total run time was 4 minutes. The flow rate was set at $0.5 \mathrm{~mL} / \mathrm{min}$ and the column temperature was maintained at $30^{\circ} \mathrm{C}$. The samples were monitored at $231 \mathrm{~nm}$ using an ultraviolet detector.

\section{Assay and Purity Test}

For assay test, the samples were prepared at approximately $0.3 \mathrm{mg} / \mathrm{mL}$ by dissolving the SD powder into the diluent and sonication could facilitate dissolving. Inject $1 \mu \mathrm{L}$ of the sample solution to a UPLC system and use the same chromatographic conditions as the section, "Miniaturized Two-Step Kinetic Solubility Test." A stability indicating method for ZL006 was developed and used for purity test, which used Waters UPLC system with a reversed phase column (Acquity UPLC BEH Shield RP18, $2.1 \mathrm{~mm} \times 100 \mathrm{~mm}, 1.7 \mu \mathrm{m}$ ) with column temperature at $30^{\circ} \mathrm{C}$. The mobile phase consisted of $95 \%$ $10 \mathrm{mmol} / \mathrm{L}$ ammonia acetate buffer $(\mathrm{pH} 7.0)$ and 5\% acetonitrile (A)/95\% acetonitrile and $5 \%$ water (B), the gradient of $\mathrm{A}(\%)$ was $98,35,0,0,98$ at $0,10,12,13$, and 13.1 minutes, respectively, and total run time was 15 minutes. The flow rate was set at $0.5 \mathrm{~mL} / \mathrm{min}$ and the samples were monitored at $231 \mathrm{~nm}$ using an ultraviolet detector. The preparation of sample solution was the same as for the assay test.

\section{X-Ray Powder Diffraction}

The physical form of the compound and SD formulations were evaluated by XRPD (D8 Advance, Bruker, United States) 
using $\mathrm{Cu} K_{\alpha}$ radiation at $40 \mathrm{kV}$ generator voltage and $40 \mathrm{~mA}$ current with a scan range of 5 to 45 degrees $(2 \theta)$ at a scanning rate of 2-degree per minute.

\section{Thermal Analysis (Differential Scanning Calorimetry,} DSC)

Thermal analysis of the SDs was conducted by DSC (Q2000, TA Instrument, New Castle, Delaware, United States). From 1 to $5 \mathrm{mg}$ of sample was sealed in an aluminum pan with an empty pan for reference. For modulated DSC (mDSC), modulation at $\pm 1^{\circ} \mathrm{C}$ at every 60 seconds and ramping at $2^{\circ} \mathrm{C} / \mathrm{min}$ were used.

\section{Fourier's Transform Infrared (FTIR) Spectroscopy}

FTIR spectra of ZL006, Soluplus, HPMCAS-LF (Hydroxypropyl methylcellulose-acetate succinate-LF), PM, and SDs obtained by HME were collected using an FTIR spectrometer (Nicolet 6700 FT-IR, Thermo Fisher Scientific, Waltham, Massachusetts, United States). A small amount of the sample was used, and the spectra were collected from 400 to $4,000 \mathrm{~cm}^{-1}$, with 32 scans and a resolution of $4 \mathrm{~cm}^{-1}$.

\section{In vitro Microdissolution Study}

To save the consumption of the compound, dissolution of the SD was conducted on a micro dissolution device $\mu$ Disso (Pion Inc., Billerica, Massachusetts, United States). ${ }^{2}$ Briefly, $20 \mathrm{~mL}$ of $0.5 \%$ SLS in $\mathrm{pH} 6.8$ phosphate buffer as the dissolution media at $37^{\circ} \mathrm{C}$ and $75 \mathrm{r} / \mathrm{min}$ stirring rate were used for the dissolution studies $(n=3)$. About $5 \mathrm{mg}$ of the solid dispersion or its corresponding PM (equivalent to $0.5 \mathrm{mg}$ ZL006) or $0.5 \mathrm{mg}$ of the neat compound was added to the media. The samples $(200 \mu \mathrm{L})$ were withdrawn at predetermined time points and $200 \mu \mathrm{L}$ of the dissolution media was refilled. After filtration by centrifugation, as the same method as solubility test, the concentration of ZL006 was determined by UPLC assay test method.

\section{Preliminary in vivo Study}

To evaluate the SD in vivo performance, the SD powder was prepared as a suspension in $\mathrm{pH} 6.8$ phosphate buffer containing $0.5 \%$ HPMC-E3 and then orally administered to rats. Plasma samples were taken at $0.5,1,2,4,6,8,10,24,48,96$, and 144 hours following the dosing. Plasma exposures obtained from the SD formulation were calculated and compared with the crystalline suspension composed of CMC-Na and Tween 80.

\section{Results and Discussion}

\section{SD Formulation Screening by Solvent Evaporation}

To enable effective and efficient development of SD for early stage research compound, several studies reported miniaturized method for polymer screening of solid dispersion. 2,14,18-20 In this study, solvent evaporation method followed by miniaturized two-step kinetic solubility was employed to facilitate the polymer screening with minimal consumption of the compound which is a key element in SD formulation. This method allows quick identification of the most promising polymers that could improve the kinetic solubility in biorele-

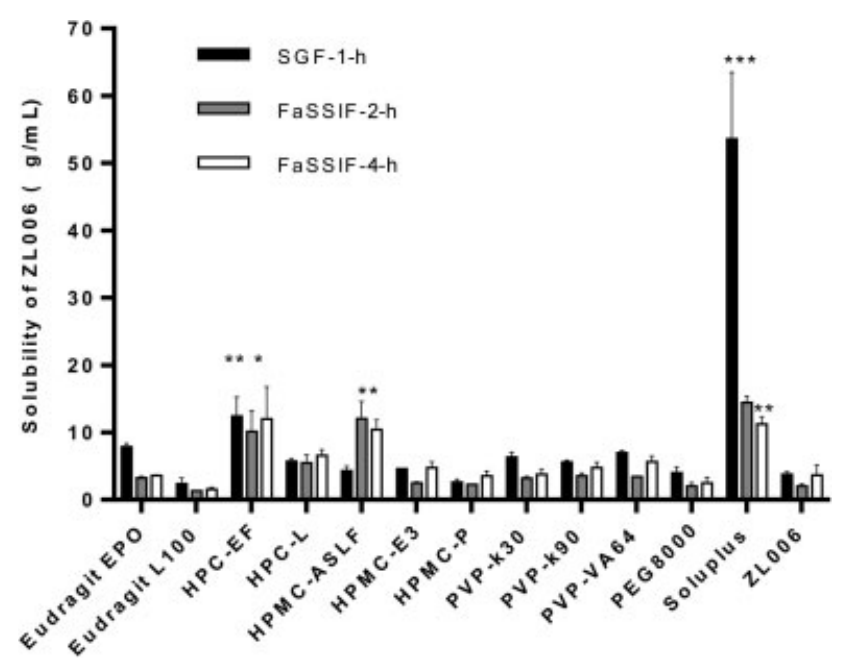

Fig. 2 Two-step kinetic solubilities of ZL006 in biorelevant media obtained from solid dispersions containing different polymers at $20 \%$ drug load $(n=3)$. $^{*}: p<0.05,{ }^{* *}: p<0.01,{ }^{* * *}: p<0.001$ vs. ZL006 group. FaSSIF, fasted simulated small intestinal fluid; HPMCAS-LF, hydroxypropyl methylcellulose acetate succinate-LF; SGF, simulated gastric fluid; Eudragit EPO, Eudragit E powder; HPC-EF, hydroxypropyl cellulose-EF; HPC-L, hydroxypropyl cellulose-L; HPMC-E3, hydroxypropyl methyl cellulose-E3; HPMC-P, hydroxypropyl methyl cellulosephthalate; PVP, polyvinylpyrrolidone, PEG, polyethylene glycol.

vant media. The miniaturized two-step kinetic solubility also mimics the transition of a compound in vivo from gastric to small intestine. And solubilities at different time points were measured to assess the potential of a SD to reach a supersaturated concentration and the ability to maintain the supersaturation state which is critical for its rapid absorption and enhanced bioavailability. The remaining SD sample can be used for XRPD characterization which could provide the ranking order of the recrystallization inhibition effect of each polymer.

The kinetic solubility of ZL006 for each SD at 20\% drug load is presented in - Fig. 2 . Soluplus was observed to improve the solubility in the first hour in SGF remarkably with statistical significance $(p<0.001)$; however, the kinetic solubility dropped dramatically at 2- and 4-hour time points in fasted simulated small intestinal fluid (FaSSIF), indicating that it might not be able to maintain the supersaturation. The second-tier polymers were HPC-EF and HPMCAS-LF, both showing similar improved solubilities in FaSSIF at 2 and 4 hours with statistical significance compared with ZL006 ( $p<0.05$ for HPC-EF and $p<0.01$ for HPMCAS-LF), but the SD with HPMCAS-LF showed lower solubility in SGF which did not statistically differ from the pure compound $(p>0.05)$, mainly, because the polymer itself only dissolves in $\mathrm{pH}$ above 5.5. Considering HPMCAS-LF was not dissolved in SGF, the polymer may hinder the solubilization of the compound dispersed in it. In comparison, another enteric polymer hydroxypropyl methylcellulose phthalate (HPMC-P) did not show any solubility improvement in FaSSIF (no statistically significant difference compared with the pure compound, $p>0.05$ ), indicating HPMCAS-LF played some role in the solubility improvement of ZL006. Then the three SDs with the polymers of most interests were detected 


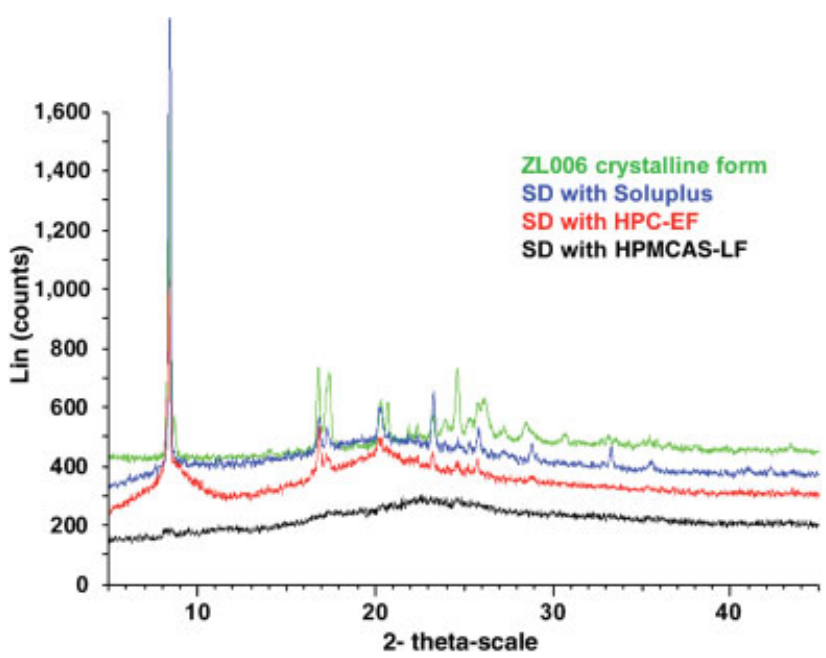

Fig. 3 XRPD patterns of SDs with different polymers by solvent evaporation at $20 \%$ drug load. SD, solid dispersion; Vit-E-TPGS, vitamin-E-tocopheryl polyethylene glycol 1,000 succinate; XRPD, X-ray powder diffraction.

by XRPD and the XRPD patterns are presented in - Fig. 3. After solvent evaporation, SDs with HPC-EF and Soluplus still showed ZL006 characteristic peaks in the XRPD patterns, but with lower crystallinity compared with ZL006 initial form, indicating the compound was not transformed to amorphous state completely. While the formulation with HPMCAS-LF was amorphous, indicating HPMCAS-LF had better crystallization inhibition effect compared with the other two polymers which was favorable for the solubility improvement, supersaturation maintenance, and SD stability. The parachute effect of HPMCAS that could strongly inhibit crystallization of drug in supersaturated solutions was also reported in other studies. ${ }^{16,21-23}$

The equilibrium solubilities of ZL006 in different surfactant solutions are presented in - Fig. 4. SLS and vitamin-Etocopheryl polyethylene glycol 1,000 succinate (TPGS) were

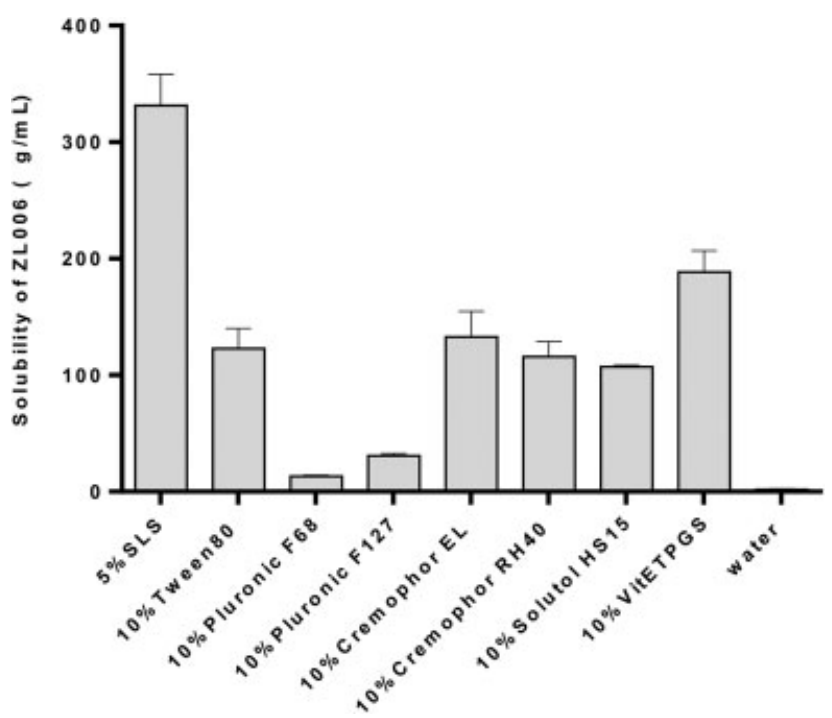

Fig. 4 Equilibrium solubilities of ZL006 in different surfactants solutions $(n=3)$. SLS, Sodium lauryl sulfate.

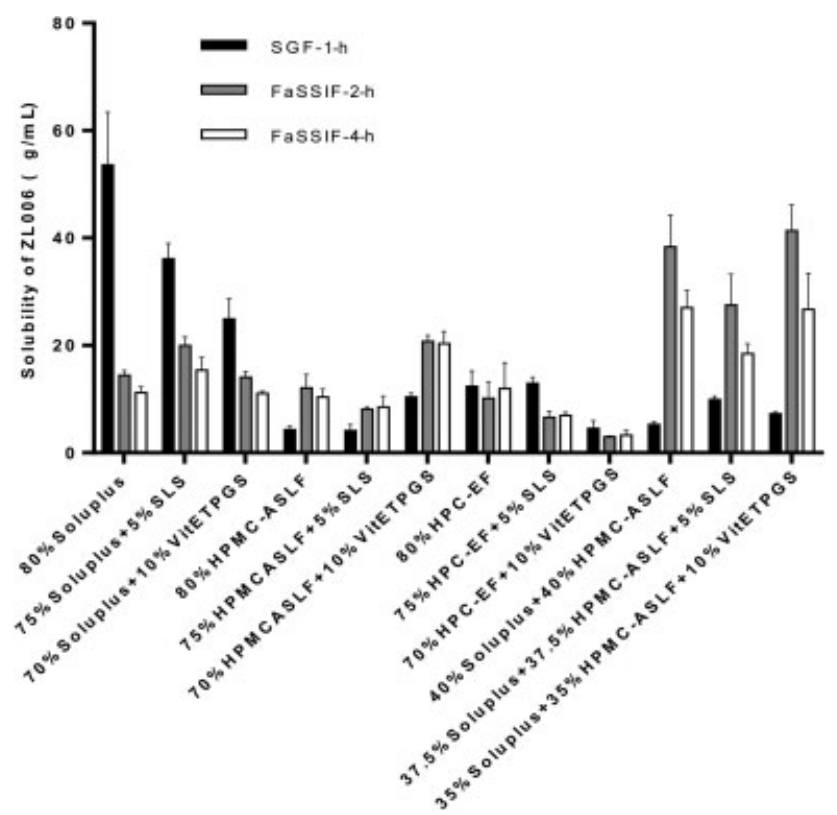

Fig. 5 Two-step kinetic solubilities of ZL006 in biorelevant media obtained from different SD formulations at $20 \%$ drug load $(n=3)$. FaSSIF, fasted simulated small intestinal fluid; SD, solid dispersion; SGF, simulated gastric fluid; SLS, Sodium lauryl sulfate; Vit-E-TPGS, vitamin-E-tocopheryl polyethylene glycol 1,000 succinate.

identified as the two most promising surfactants to improve the solubility of ZL006.

Based on the identified three polymers and two surfactants, different SD formulation variants were prepared by solvent evaporation method and the kinetic solubility results of different variants are presented in $\mathbf{- F i g .}$ 5. SDs containing HPC-EF with either surfactant showed lower solubility than that without surfactants, indicating HPC-EF was the major contributor of solubility enhancement among these three excipients. The similar trend was also observed in formulations containing Soluplus. In comparison, the formulation containing HPMCASLF and vitamin-E-TPGS showed higher solubility at all three time points than the SD with HPMCAS-LF only, indicating vitamin-E-TPGS may help the solubilization of the compound in SGF, thus further facilitating HPMCAS-LF to play its role in FaSSIF. Considering the formulations containing HPC-EF did not show any advantage in solubility improvement, this polymer was ruled out for further studies.

Interestingly, with the combination of Soluplus and HPMCAS-LF, all the three formulations showed much higher solubility in FaSSIF at 4-hour compared with all the others, though solubilities in SGF were still low. As previous study demonstrated that the main absorption site for ZL006 was small intestine, ${ }^{24}$ it suggested that solubility enhancement in FaSSIF would play more critical role in its absorption maximization than solubility increase in SGF. Based on the above kinetic solubility results, it was speculated that Soluplus helped ZL006 to reach supersaturated solubility, while HPMCAS-LF maintained its supersaturation in FaSSIF. Instead of a single polymer, the polymer combinations could take advantage of each individual polymer's properties to improve the SD performance. Many studies discussed the SD formulations composed 
of polymer combinations with the purpose to improve solubility of poorly soluble compounds and meanwhile to enhance the stability of the amorphous SD. $8,25,26$ The combination of Soluplus and HPMCAS was also reported for other insoluble compounds. ${ }^{27,28}$ In this case, the solubility screening indicated the use of Soluplus and HPMCAS-LF blends. Taking HME process and SD stability into consideration, this combination would also have advantages over single polymer. The glass transition temperature $\left(T_{\mathrm{g}}\right)$ values of Soluplus and HPMCAS-LF are 70 and $120^{\circ} \mathrm{C}$, respectively. On one hand, the SDs with single Soluplus would not have high $T_{\mathrm{g}}$ which is not favorable for its physical stability, though the low $T_{\mathrm{g}}$ of Soluplus is ideal for HME process; on the other hand, SDs with single HPMCAS-LF would not be suitable for HME process due to its high $T_{\mathrm{g}}$, but incorporation of polymer with high $T_{\mathrm{g}}$ could improve physical stability of the SDs. Thus, the combination of Soluplus and HPMCAS-LF would not only maximize the solubility improvement but also facilitate the extrudability and increase the $T_{\mathrm{g}}$ of SDs which would subsequently enhance the SDs physical stability. With respect of surfactants, there are also two contradictory aspects. On one hand, surfactants are capable of improving wettability and solubility which are generally used as plasticizers to increase processability for HME process; on the other hand, surfactants may decrease the $T_{\mathrm{g}}$ of extruded SDs and have negative impact on the physical stability. Therefore, the need of incorporation of vitamin-E-TPGS into the SD formulation will be investigated by HME process. Take into account of all the above mentioned, the lead SD formulations containing Soluplus and HPMCAS-LF combination with and without vitamin-E-TPGS were identified and will be further optimized by HME process.

\section{Optimization and Scale up of Lead SD Formulation by HME}

Optimization of Formulation and Process Parameters by Orthogonal Design

Several preliminary trials have been made with the lead SD formulations by HME. It was observed that incorporation of vitamin-E-TPGS into the formulation could make HME process more smooth and the extruded SDs showed higher solubility than that without vitamin-E-TPGS. Meanwhile, the $T_{\mathrm{g}}$ of the SDs with vitamin-E-TPGS was approximately $70^{\circ} \mathrm{C}$. Based on " $T_{\mathrm{g}}-50$ rule" ${ }^{4,8}$ which indicates the storage temperature usually should be around $50^{\circ} \mathrm{C}$ below the $T_{\mathrm{g}}$, the SD would be theoretically physically stable at around room temperature, though the $T_{\mathrm{g}}$ was approximately $15^{\circ} \mathrm{C}$ lower than the $T_{\mathrm{g}}$ of the SDs without vitamin-E-TPGS. Based on the preliminary test results, the SD formulation composed of Soluplus, HPMCAS-LF and vitamin-ETPGS was further optimized by orthogonal design. Totally nine formulations were prepared by HME with different levels of drug load, process temperature, and screw speed as shown in - Table 1. The summary of characterization and the two-step kinetic solubility for the nine formulations are presented in -Table 2 and -Fig. 6, respectively. The results of ANOVA for each factor's impact on the solubility at different time points are listed in -Table 3. From characterization results, it was observed that with the increase of drug load, the extrudates color became darker and the transparency decreased, as the starting material was yellow. Transparency was consistent to XRPD pattern, as small ZL006 characteristic peaks were observed in XRPD pattern for all three formulations at $20 \%$ drug load which were not transparent at all; indicating regardless of the HME process tested the compound could not be converted to amorphous state completely at drug load of $20 \%$. In comparison, formulations at lower drug load were all amorphous and transparent. HME temperature and screw speed are related to the homogeneous dispersion of the compound in the molten polymer phase, residence time, and shear force, which subsequently have impacts on the solubility and purity of the extrudates. The purities of ZL006 were below $99 \%$ when the extrudates were prepared at $180^{\circ} \mathrm{C}$, while the purities were all above $99 \%$ at lower temperatures and significant degradation was observed for ZL006 when HME temperature was set at $190^{\circ} \mathrm{C}$ during pretest before optimization test.

The overall solubility at three time points were higher at $10 \%$ drug load compared with those of higher drug load irrespective of HME process parameters. All formulations showed relatively low solubility in SGF and none of the three factors showed significant effect on the solubility in SGF at

Table 2 Summary of characterization for the nine SD formulations by orthogonal design $(n=3)$

\begin{tabular}{|l|l|l|l|l|l|}
\hline No. & $\begin{array}{l}\text { Drug Load, process } \\
\text { temperature and } \\
\text { screw speed }\end{array}$ & Appearance & XRPD & $\begin{array}{l}\text { Purity } \\
\text { (peak area/\%) }\end{array}$ & $\begin{array}{l}\text { Actual drug } \\
\text { load/\% }\end{array}$ \\
\hline 1 & $10 \%, 160^{\circ} \mathrm{C}, 100 \mathrm{rpm}$ & Orange, transparent & Amorphous & $99.18 \pm 0.12$ & $10.29 \pm 0.16$ \\
\hline 2 & $10 \%, 170^{\circ} \mathrm{C}, 150 \mathrm{rpm}$ & Orange, transparent & Amorphous & $99.27 \pm 0.04$ & $9.48 \pm 0.18$ \\
\hline 3 & $10 \%, 180^{\circ} \mathrm{C}, 200 \mathrm{rpm}$ & Orange, transparent & Amorphous & $98.94 \pm 0.02$ & $10.09 \pm 0.04$ \\
\hline 4 & $15 \%, 160^{\circ} \mathrm{C}, 150 \mathrm{rpm}$ & Dark orange, transparent & Amorphous & $99.49 \pm 0.05$ & $14.91 \pm 0.22$ \\
\hline 5 & $15 \%, 170^{\circ} \mathrm{C}, 200 \mathrm{rpm}$ & Dark orange, transparent & Amorphous & $99.47 \pm 0.03$ & $13.66 \pm 0.23$ \\
\hline 6 & $15 \%, 180^{\circ} \mathrm{C}, 100 \mathrm{rpm}$ & Dark orange, transparent & Amorphous & $98.49 \pm 0.08$ & $14.65 \pm 0.17$ \\
\hline 7 & $20 \%, 160^{\circ} \mathrm{C}, 200 \mathrm{rpm}$ & Dark orange, not transparent & Low crystalline & $99.68 \pm 0.01$ & $19.33 \pm 0.50$ \\
\hline 8 & $20 \%, 170^{\circ} \mathrm{C}, 100 \mathrm{rpm}$ & Dark orange, not transparent & Low crystalline & $99.49 \pm 0.01$ & $20.95 \pm 0.84$ \\
\hline 9 & $20 \%, 180^{\circ} \mathrm{C}, 150 \mathrm{rpm}$ & Brown, not transparent & Low crystalline & $98.05 \pm 0.02$ & $19.65 \pm 0.28$ \\
\hline
\end{tabular}

Abbreviations: SD, solid dispersion; XRPD, X-ray powder diffraction. 


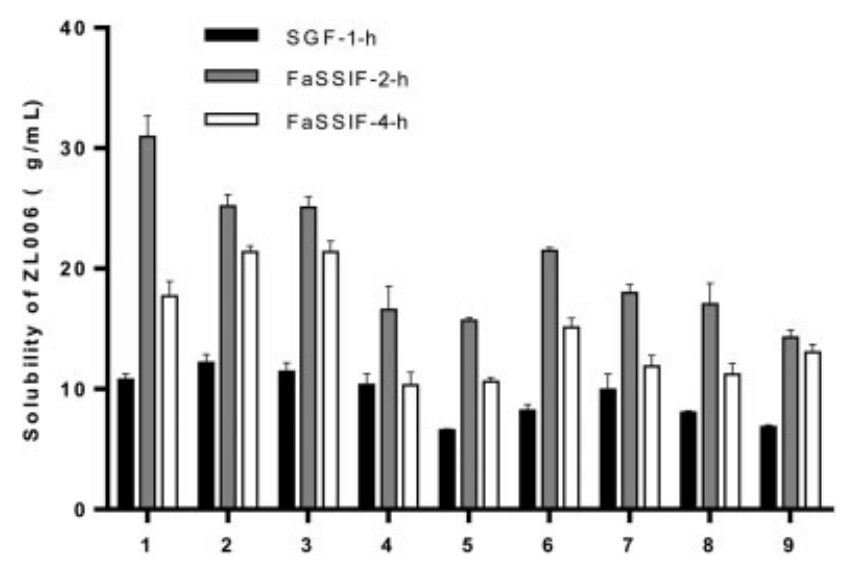

Fig. 6 Two-step kinetic solubilities of ZL006 in biorelevant media obtained from different SD formulations by HME with orthogonal design $(n=3)$. FaSSIF, fasted simulated small intestinal fluid; HME, hot-melt extrusion; SD, solid dispersion; SGF, simulated gastric fluid.

1 hour statistically $(p>0.05)$, mainly because HPMCAS did not dissolve in SGF which hindered the solubilization of the compound. The drug load showed statistically significant effect ( $p<0.01$ ) on the solubility in FaSSIF at both time points. The solubilities in FaSSIF were the highest at 10\% drug load and decreased with the increase of drug load irrespective of HME process parameters. In comparison, neither HME temperature nor the screw speed showed statistical significance on the solubilities in both SGF and FaSSIF. The theoretical prediction and calculation of the glass-forming ability and crystallization tendency of ZL006 is 1.45 which is defined as the reciprocal of $T_{\mathrm{g}} / T_{\mathrm{m}}$ (both in Kelvin's temperature, the $T_{\mathrm{g}}$ and $T_{\mathrm{m}}$ of ZL006 is 68 and $220^{\circ} \mathrm{C}$, respectively). The $T_{\mathrm{m}} / T_{\mathrm{g}}$ value of 1.45 indicated the compound was not easy to get glassy state and had higher tendency to crystallize; drug load for the compounds with $T_{\mathrm{m}} / T_{\mathrm{g}}$ value ranging from 1.4 to 1.6 is usually low. ${ }^{16,22,29}$ This is consistent with the optimization results, with the drug load above $10 \%$, the solubility could not be enhanced by elevated temperature and screw speed change, and the crystalline compound could not be converted to amorphous completely when drug load was increased to $20 \%$ even at $180^{\circ} \mathrm{C}$.

Though the mini-extruder saves the amount of compound, the miniaturization of the equipment is at the expense of shorter residence time at molten status due to shorter screws and lack of some important functions compared with larger-scale extruder, such as multiple elements for screws. The mini-extruder applied here has no kneading element that usually provides main shear force, and only has conveying element for the screw. This simple screw configuration could also be the reason for the low-drug load, as the shear force might not be sufficient for the compound with

Table 3 One-way analysis of variance (ANOVA) of the solubility by the orthogonal design

\begin{tabular}{|c|c|c|c|c|c|c|}
\hline \multicolumn{7}{|l|}{ SGF-1-h } \\
\hline Factor & Source & Sum of squares & DF & F-ratio & $p$-Value & Significance \\
\hline \multirow[t]{2}{*}{ Drug load } & Drug load & 19.63 & 2 & 4.556 & 0.0626 & $*$ \\
\hline & Error & 12.93 & 6 & & & \\
\hline \multirow[t]{2}{*}{ Temperature } & Temperature & 4.374 & 2 & 0.4647 & 0.6492 & $*$ \\
\hline & Error & 28.24 & 6 & & & \\
\hline \multirow[t]{2}{*}{ Screw speed } & Screw speed & 0.9142 & 2 & 0.0865 & 0.9182 & $*$ \\
\hline & Error & 31.7 & 6 & & & \\
\hline \multicolumn{7}{|l|}{ FaSSIF-2-h } \\
\hline \multirow[t]{2}{*}{ Drug load } & Drug load & 199.4 & 2 & 12.06 & 0.0079 & $* *$ \\
\hline & Error & 49.6 & 6 & & & \\
\hline \multirow[t]{2}{*}{ Temperature } & Temperature & 239.2 & 2 & 0.1218 & 0.8874 & * \\
\hline & Error & 28.24 & 6 & & & \\
\hline \multirow[t]{2}{*}{ Screw speed } & Screw speed & 33.88 & 2 & 0.4725 & 0.6448 & $*$ \\
\hline & Error & 215.1 & 6 & & & \\
\hline \multicolumn{7}{|l|}{ FaSSIF-4-h } \\
\hline \multirow[t]{2}{*}{ Drug load } & Drug load & 132.3 & 2 & 15.83 & 0.004 & $* *$ \\
\hline & Error & 25.07 & 6 & & & \\
\hline \multirow[t]{2}{*}{ Temperature } & Temperature & 15.87 & 2 & 0.3365 & 0.7269 & $*$ \\
\hline & Error & 141.5 & 6 & & & \\
\hline \multirow[t]{2}{*}{ Screw speed } & Screw speed & 0.166 & 2 & 0.0032 & 0.9968 & $*$ \\
\hline & Error & 157.2 & 6 & & & \\
\hline
\end{tabular}

Abbreviations: DF, degree of freedom; FaSSIF, fasted simulated small intestinal fluid; SGF, simulated gastric fluid.

${ }^{*} p>0.05$.

${ }^{* *} p<0.01$. 


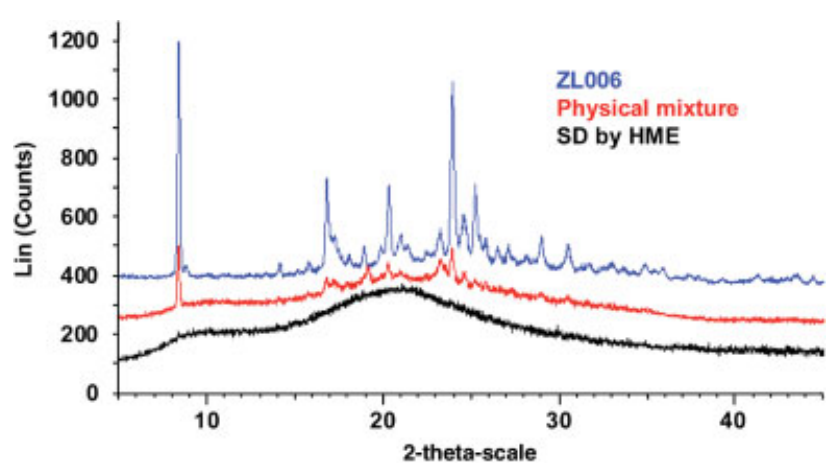

Fig. 7 XRPD patterns of the SDs and physical mixture. HME, hot-melt extrusion; SD, solid dispersion; XRPD, X-ray powder diffraction.

higher tendency to crystallize. Nevertheless, this miniaturized HME still served the purpose to develop a prototype SD for the challenging molecule ZL006. Considering lower temperature is preferred for compound chemical stability and lower screw speed extends the residence time to facilitate the homogeneous dispersion, the optimal SD formulation was 10\% ZL006, 40\% Soluplus, 40\% HPMCAS-LF, and 10\% vitamin-E-TPGS prepared by HME at $160^{\circ} \mathrm{C}$ and $100 \mathrm{r} / \mathrm{min}$ screw speed.

\section{Scale up of the Optimal Formulation by HME}

The SD formulation composed of 10\% ZL006, 40\% Soluplus, 40\% HPMCAS-LF, and 10\% vitamin-E-TPGS was scaled up by HME with process temperature at $160^{\circ} \mathrm{C}$ and screw speed was $100 \mathrm{r} / \mathrm{min}$ at batch size of $18 \mathrm{~g}$. The process was smooth and the extrudates were transparent orange, brittle, and easy to mill. Approximately $16 \mathrm{~g}$ of SD powder (89\% yields) was finally obtained and the purity of ZL006 was $99.34 \%$.

\section{Characterization of the Scale up SD}

XRPD was utilized to differentiate crystalline state and amorphous state. ZL006 showed obvious diffraction peaks at 8.5, 17, 20, and 24 degrees in - Fig. 7. The PM showed the characteristic peaks of ZL006, but with lower crystallinity, due to the low-drug content of $10 \%$. In comparison, the diffractogram of SDs prepared by HME did not show any peaks, but only a big halo, indicating the compound was converted from crystalline form to amorphous form after HME process.

mDSC was performed to test the $T_{\mathrm{g}}$ of the SDs and also compared with PM to further confirm the state of the compound in SD. In the mDSC thermogram of PM ( - Fig. 8A), four thermal events were observed, including the first sharp endothermic peak corresponding to the melting of vitamin-E-TPGS, the second and third thermal events at around 80 and $119^{\circ} \mathrm{C}$ corresponding to the $T_{\mathrm{g}}$ of Soluplus and HPMCAS-LF, respectively, and the final small melting peak indicating the crystalline compound. By contrast, in - Fig. 8B, the SDs prepared by HME showed a single $T_{\mathrm{g}}$ at $69^{\circ} \mathrm{C}$, indicating the compound and excipients were intimately mixed to a single phase with amorphous state after HME process.

IR spectrum of the SD sample prepared by HME is shown in -Fig. 9, with the intention to identify the intermolecular interactions between the compound and the excipients that play important roles in the formation, crystallization inhibition, and stabilization of SD. ${ }^{4}$ The spectrum of ZL006 shows characteristic peak at $1659 \mathrm{~cm}^{-1}$ corresponding to $\mathrm{C}=\mathrm{O}$ stretch vibration in its structure. Soluplus has characteristic peaks corresponding to $\mathrm{C}-\mathrm{H}$ stretch at 2,925 and $2,857 \mathrm{~cm}^{-1}$ and two strong sharp peaks at 1,731 and $1,627 \mathrm{~cm}^{-1}$ corresponding to $\mathrm{C}=\mathrm{O}$ stretch bending from ester group and caprolactam ring, respectively. ${ }^{30}$ HPMCAS has strong hydrogen donor, which is attributed to its $\mathrm{O}-\mathrm{H}$ stretch peak at around $3,400 \mathrm{~cm}^{-1}$. 21,31 The distinct peaks of ZL006 and HMPCAS-LF were observed to be unchanged in the spectrum of the PM, indicating few interactions occur between them when mixed physically. In comparison, the characteristic peak of ZL006 at $1,659 \mathrm{~cm}^{-1}$ shows significant shift to $1,691 \mathrm{~cm}^{-1}$ in the SDs sample, indicating the hydrogen bonding formation between a hydrogen acceptor $(\mathrm{C}=\mathrm{O})$ of the compound and a hydrogen donor $(\mathrm{O}-\mathrm{H})$ from HPMCAS-LF.

The two-step kinetic solubility of SDs was compared with the corresponding PM and crystalline pure compound, which is shown in -Fig. 10. The solubility results of the scale-up SD were similar to its screening results, and were much higher than PM, mainly due to its supersaturation state by amorphous SDs, while PM showed higher solubility than the pure compound because of the solubilization and wettability improvement by surfactant and Soluplus.

\section{In vitro Dissolution}

A non-sink condition dissolution was applied to compare the dissolution profiles of the SDs, PM, and the pure compound. To save the amount of the compound, a microdissolution apparatus was employed, and the dissolution profiles were presented in - Fig. 10. The compound was not wettable and had extremely poor solubility in pH 6.8 buffer (below LOD), so 0.5\% SLS was added to pH 6.8 buffers as the dissolution media based on the solubility study. The dissolution profile showed that around $12 \%$ of crystalline ZL006 dissolved at 15 minutes and 34\% dissolved at 60 minutes. The PM increased dissolution rate compared with the pure compound, which could be due to solubilization effect of vitamin-E-TPGS and Soluplus in the mixture. In contrast to PM, SDs prepared by HME exhibited remarkable faster and complete dissolution. Combined with the characterization results, it was assumed that the improved dissolution rate could be attributed to the amorphous state that achieved supersaturation and other factors, such as the increased wettability, greater hydrophilicity, improved dispersibility, and reduction in particle size of the compound.

\section{Preliminary in vivo Evaluation of SDs by HME}

A preliminary pharmacokinetics (PK) study in rats was conducted for the SDs and was compared with the PK results obtained from ZL006 suspension after oral administration. The PK parameters are summarized in - Table 4. - Fig. 11 provides the mean plasma concentrations of ZL006 after a single oral dose of SDs at $12.5 \mathrm{mg} / \mathrm{kg}(\mathrm{A})$, and suspension (B) at $10 \mathrm{mg} / \mathrm{kg}$. As the AUC (area under curve) between the SD and the suspension differed substantially, the PK profiles are presented separately. The dose normalized AUC of the SD was approximately 230-fold higher than that of the suspension, indicating 

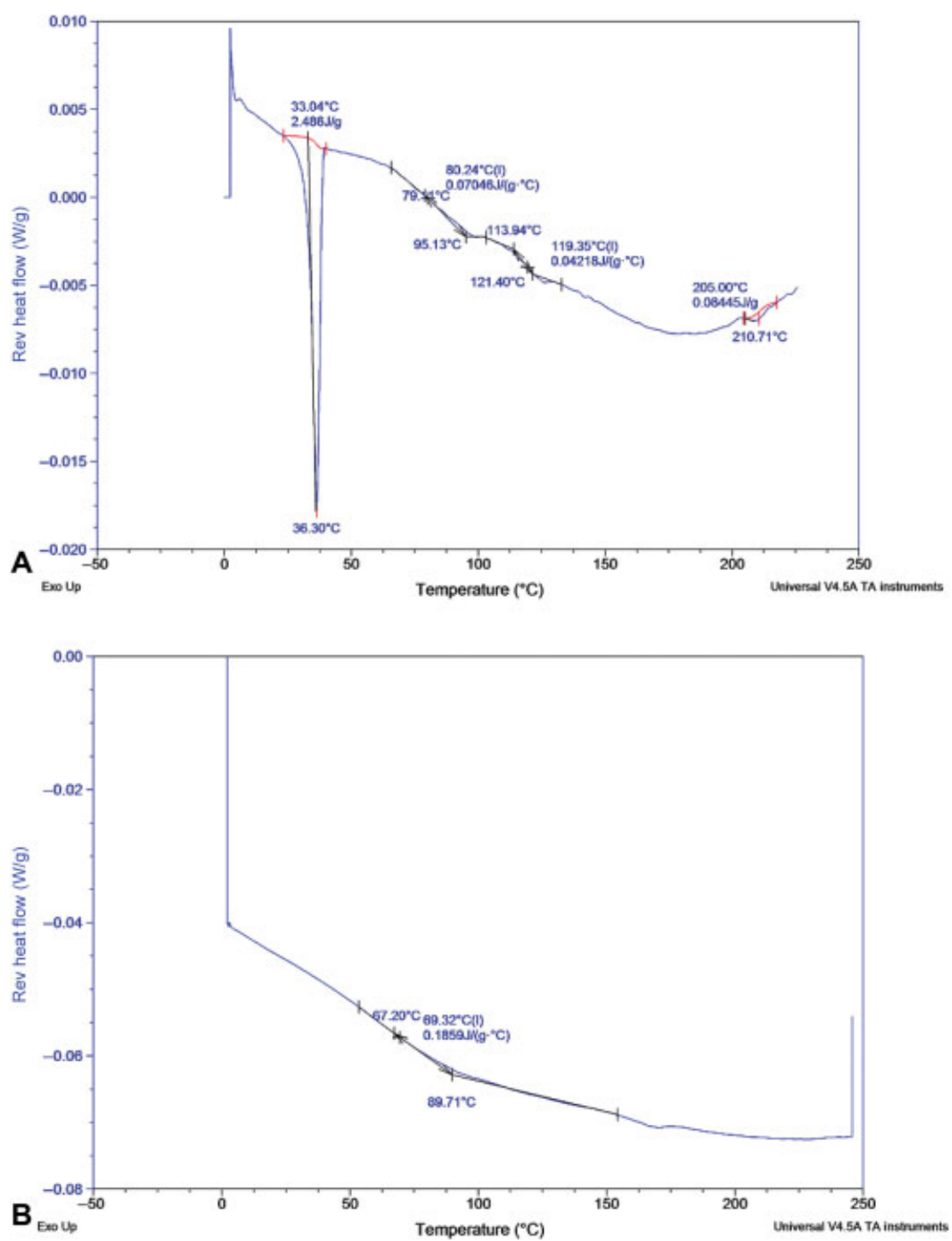

Fig. 8 mDSC patterns of physical mixture (A) and SDs by HME (B). mDSC, modulated differential scanning calorimetry; HME, hot-melt extrusion; $\mathrm{PM}$, physical mixture; SD, solid dispersion.

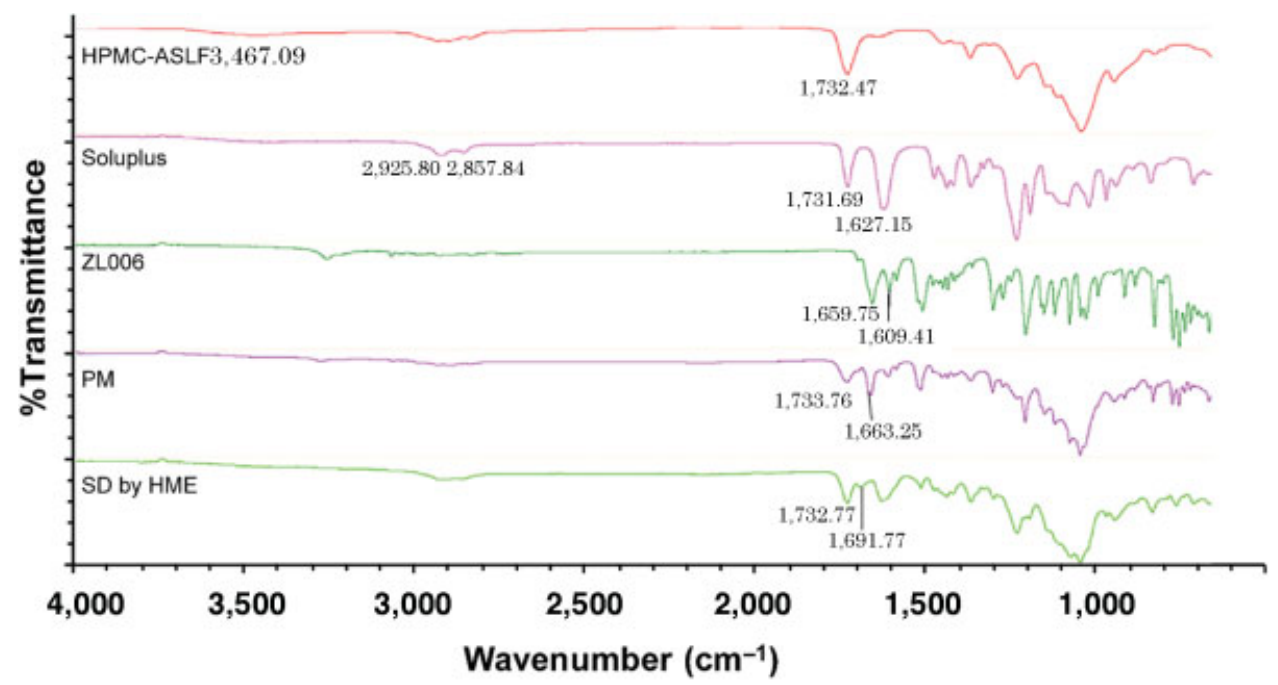

Fig. 9 FTIR spectra of the excipients, compound, PM and SDs. FTIR, Fourier's transform infrared spectroscopy; HME, hot-melt extrusion; HPMCAS-LF, hydroxypropyl methylcellulose acetate succinate-LF; SD, solid dispersion. 

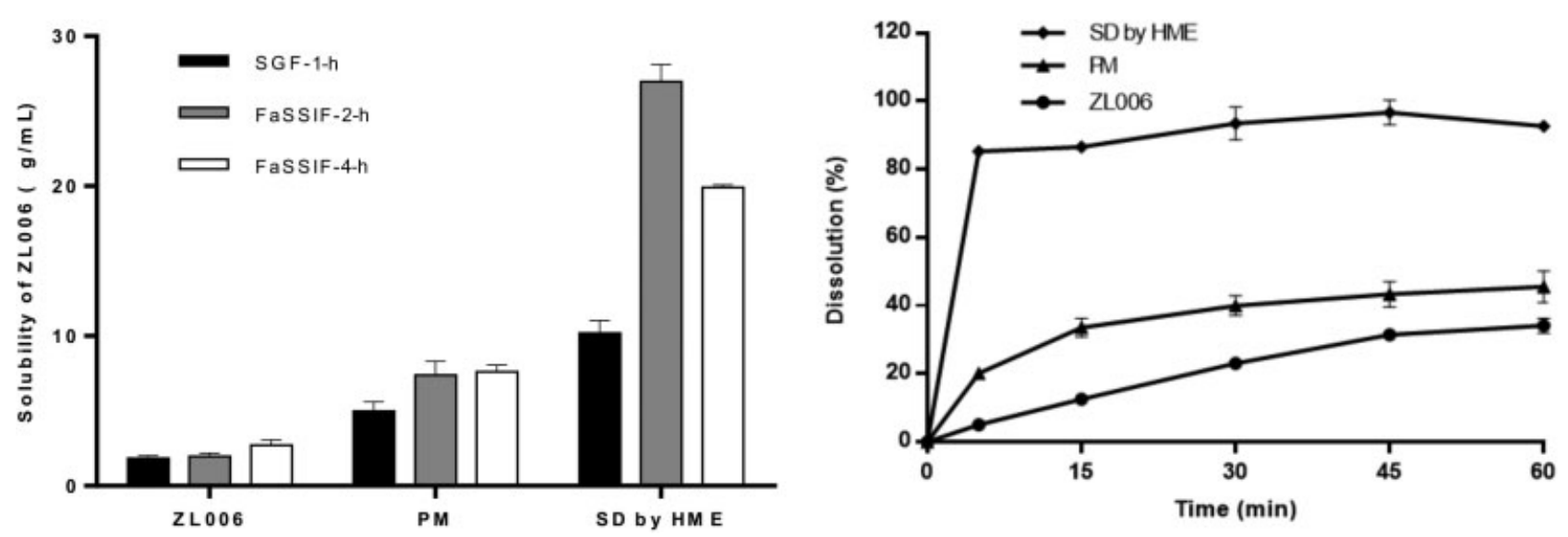

Fig. 10 Two-step kinetic solubilities (left) and dissolution profiles (right) of ZL006 from pure compound, PM, and scale up SDs by HME ( $n=3$ ). FaSSIF, fasted simulated small intestinal fluid; HME, hot-melt extrusion; PM, physical mixture; SD, solid dispersion; SGF, simulated gastric fluid.

Table 4 ZL006 PK parameters following the oral administration of listed formulations in rats $(n=6)$

\begin{tabular}{|l|l|l|}
\hline Parameters & Solid dispersion & Suspension \\
\hline Dose $/ \mathrm{mg} \cdot \mathrm{kg}^{-1}$ & 12.5 & 10 \\
\hline $\mathrm{AUC}_{0 \rightarrow \infty} / \mathrm{mg} \cdot \mathrm{h} \cdot \mathrm{L}^{-1}$ & $315.83 \pm 141.75$ & $1.10 \pm 0.12$ \\
\hline $\begin{array}{l}\mathrm{AUC} C_{0 \rightarrow \infty} / \\
\text { dose } / \mathrm{kg} \cdot \mathrm{h} \cdot \mathrm{L}^{-1}\end{array}$ & $25.27 \pm 11.34$ & $0.11 \pm 0.01$ \\
\hline$C_{\max } / \mathrm{mg} \cdot \mathrm{L}^{-1}$ & $10.47 \pm 2.05$ & $0.046 \pm 0.003$ \\
\hline$t_{\max } / \mathrm{h}$ & $8.33 \pm 1.97$ & $6.47 \pm 0.51$ \\
\hline
\end{tabular}

Abbreviations: AUC, area under curve; $t_{\max }$, the time to reach the $c_{\max }$; $c_{\max }$, the maximum drug concentration in plasma.

that the SD improved the oral bioavailability by 230 -fold to $71 \%$, as the oral bioavailability of the suspension was as low as only $0.31 \%$. Interestingly, the compound was still detected at 144 hours after oral dosing. It was suspected that possible enteric-liver circulation (though no two-peaks in AUC was

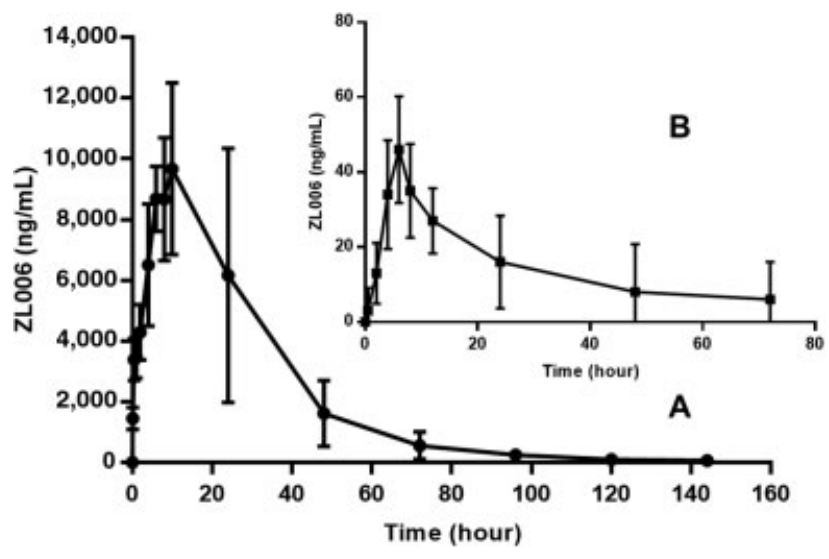

Fig. 11 ZL006 PK profiles of different formulations (A for SDs, B for suspension) in rats $(n=6)$. (A) mean plasma concentration of ZL006 after oral administration of the SDs at $12.5 \mathrm{mg} / \mathrm{kg}$; (B) mean plasma concentration of ZL006 after oral administration of the suspension at $10 \mathrm{mg} / \mathrm{kg}$. observed), delayed $t_{\max }$ and long MRT (approximately 24 hours) contributed to the high AUC. Moreover, the permeability of ZL006 was moderate $\left(P_{\text {app }}\right.$ was $1.95 \times 10^{-6} \mathrm{~cm} / \mathrm{s}$ on Caco-2 cell) and efflux was observed; thus, the remarkable improvement in the oral absorption indicated that the SDs might not only enhance the solubility but also increase the permeability. It was reported that Soluplus showed strong effect on the enhancement of insoluble drugs flux across Caco2 monolayer with the SD formulation containing Soluplus. ${ }^{32,33}$ Vitamin-E-TPGS is also well known for its solubilization effect and permeability enhancement. ${ }^{34}$ It should be noted that the hypothesis of permeability enhancement by the solid dispersion remains further investigation. Nevertheless, the preliminary PK study demonstrated that the SDs prepared by miniaturized HME could be recommended as a promising formulation strategy to increase oral bioavailability of ZL006 to support preclinical studies.

\section{Conclusions}

ZL006 SDs were successfully developed with significantly improved solubility and bioavailability in rats. For an active molecule at preclinical stage with limited availability, solvent evaporation method, miniaturized two-step kinetic solubility test, and solid-state characterization tools were implemented to identify the lead formulation containing Soluplus, HPMCAS-LF, and vitamin-E-TPGS. This lead SD formulation was further optimized using miniaturized HME with design of experiments. Although it was challenging to convert crystalline ZL006 to amorphous state, and there are limitations for the miniaturized extruder, an optimal amorphous SD was developed with confirmation by solid-state characterization. And the preliminary PK study demonstrated that SDs could be an effective approach for ZL006 to enhance its oral bioavailability. SD development by miniaturized HME is an efficient and effective strategy for insoluble compound at early development stage by saving resources and time which provides a prototype SD formulation for further development if necessary. This strategy could also be the reference for other poorly-soluble compounds. 


\section{Conflict of Interest}

The authors report no conflicts of interest. The authors alone are responsible for the content and writing of this article.

\section{Acknowledgments}

The authors would like to thank Dr. Dai Xuan and Dr. Jin Miao from China Novartis Institute for Biomedical Research for their valuable scientific discussions.

\section{References}

1 Sun HY, Li CG, Xiao L, Wang GP, Liu QH. [Effect of ZL-004 on raising leukocyte count]. Yao Xue Xue Bao 2010;45(06):797-800

2 Zheng W, Jain A, Papoutsakis D, Dannenfelser RM, Panicucci R, Garad S. Selection of oral bioavailability enhancing formulations during drug discovery. Drug Dev Ind Pharm 2012;38(02):235-247

3 Davis MT, Egan DP, Kuhs M, et al. Amorphous solid dispersions of BCS class II drugs: a rational approach to solvent and polymer selection. Chem Eng Res Des 2016;110:192-199

4 Shah S, Maddineni S, Lu J, Repka MA. Melt extrusion with poorly soluble drugs. Int J Pharm 2013;453(01):233-252

5 Crowley MM, Zhang F, Repka MA, et al. Pharmaceutical applications of hot-melt extrusion: part I. Drug Dev Ind Pharm 2007;33 (09):909-926

6 Repka MA, Battu SK, Upadhye SB, et al. Pharmaceutical applications of hot-melt extrusion: part II. Drug Dev Ind Pharm 2007;33 (10):1043-1057

7 Repka MA, Majumdar S, Kumar Battu S, Srirangam R, Upadhye SB. Applications of hot-melt extrusion for drug delivery. Expert Opin Drug Deliv 2008;5(12):1357-1376

8 Lang B, McGinity JW, Williams RO III. Hot-melt extrusion-basic principles and pharmaceutical applications. Drug Dev Ind Pharm 2014;40(09):1133-1155

9 Agrawal AM, Dudhedia MS, Zimny E. Hot melt extrusion: development of an amorphous solid dispersion for an insoluble drug from mini-scale to clinical scale. AAPS PharmSciTech 2016;17 (01):133-147

10 Repka MA, Bandari S, Kallakunta VR, et al. Melt extrusion with poorly soluble drugs - An integrated review. Int J Pharm 2018;535 (1,2):68-85

11 Thiry J, Krier F, Evrard B. A review of pharmaceutical extrusion: critical process parameters and scaling-up. Int J Pharm 2015;479 (01):227-240

12 Nakamichi K, NakanoT, Yasuura H, Izumi S, Kawashima Y. The role of the kneading paddle and the effects of screw revolution speed and water content on the preparation of solid dispersions using a twin-screw extruder. Int J Pharm 2002;241(02):203-211

13 Maddineni S, Battu SK, Morott J, Majumdar S, Murthy SN, Repka MA. Influence of process and formulation parameters on dissolution and stability characteristics of Kollidon ${ }^{\circledR}$ VA 64 hot-melt extrudates. AAPS PharmSciTech 2015;16(02):444-454

14 Vasconcelos T, Marques S, das Neves J, Sarmento B. Amorphous solid dispersions: Rational selection of a manufacturing process. Adv Drug Deliv Rev 2016;100:85-101

15 Lang B, McGinity JW, Williams RO III. Dissolution enhancement of itraconazole by hot-melt extrusion alone and the combination of hot-melt extrusion and rapid freezing-effect of formulation and processing variables. Mol Pharm 2014;11(01):186-196

16 Sotthivirat S, McKelvey C, Moser J, Rege B, Xu W, Zhang D. Development of amorphous solid dispersion formulations of a poorly watersoluble drug, MK-0364. Int J Pharm 2013;452(1,2):73-81
17 Zecevic DE, Wagner KG. Rational development of solid dispersions via hot-melt extrusion using screening, material characterization, and numeric simulation tools. J Pharm Sci 2013;102(07):2297-2310

18 Wyttenbach N, Janas C, Siam M, et al. Miniaturized screening of polymers for amorphous drug stabilization (SPADS): rapid assessment of solid dispersion systems. Eur J Pharm Biopharm 2013;84 (03):583-598

19 Shanbhag A, Rabel S, Nauka E, et al. Method for screening of solid dispersion formulations of low-solubility compounds-miniaturization and automation of solvent casting and dissolution testing. Int J Pharm 2008;351(1,2):209-218

20 Banda A, Manchanda A, Zhang W, Alba GM, Nagapudi K. Comparative assessment of miniaturized screening approaches for selection of polymers for amorphous drug stabilization. J Pharm Sci 2018;107(03):897-908

21 Zhang Q, Zhao Y, Zhao Y, et al. Effect of HPMCAS on recrystallization inhibition of nimodipine solid dispersions prepared by hot-melt extrusion and dissolution enhancement of nimodipine tablets. Colloids Surf B Biointerfaces 2018;172(172):118-126

22 Friesen DT, Shanker R, Crew M, Smithey DT, Curatolo WJ, Nightingale JA. Hydroxypropyl methylcellulose acetate succinate-based spray-dried dispersions: an overview. Mol Pharm 2008;5(06):1003-1019

23 Vo AQ, Feng X, Zhang J, Zhang F, Repka MA. Dual mechanism of microenvironmental $\mathrm{pH}$ modulation and foam melt extrusion to enhance performance of HPMCAS based amorphous solid dispersion. Int J Pharm 2018;550(1-2):216-228

24 Zhao F, Luan H, Ma Y, et al. Intestinal absorption of PLGA nanoparticles loaded with ZL004. Chinese Journal of Pharmaceutics 2014;45(01):49-55. DOI 10.16522/j.cnki.cjph.2014.01.014

25 Albadarin AB, Potter CB, Davis MT, et al. Development of stabilityenhanced ternary solid dispersions via combinations of HPMCP and Soluplus processed by hot melt extrusion. Int J Pharm 2017; 532(01):603-611

26 Zecevic DE, Meier R, Daniels R, Wagner KG. Site specific solubility improvement using solid dispersions of HPMC-AS/HPC SSL-mixtures. Eur J Pharm Biopharm 2014;87(02):264-270

27 Alshahrani SM, Lu W, Park JB, et al. Stability-enhanced hot-melt extruded amorphous solid dispersions via combinations of Soluplus and HPMCAS-HF. AAPS PharmSciTech 2015;16(04):824-834

28 Pawar J, Tayade A, Gangurde A, Moravkar K, Amin P. Solubility and dissolution enhancement of efavirenz hot melt extruded amorphous solid dispersions using combination of polymeric blends: A QbD approach. Eur J Pharm Sci 2016;88(88):37-49

29 He Y, Ho C. Amorphous solid dispersions: utilization and challenges in drug discovery and development. J Pharm Sci 2015;104 (10):3237-3258

30 Penumetcha SS, Gutta LN, Dhanala H, et al. Hot melt extruded Aprepitant-Soluplus solid dispersion: preformulation considerations, stability and in vitro study. Drug Dev Ind Pharm 2016;42 (10):1609-1620

31 Lu J, Obara S, Liu F, Fu W, Zhang W, Kikuchi S. Melt extrusion for a high melting point compound with improved solubility and sustained release. AAPS PharmSciTech 2018;19(01):358-370

32 Linn M, Collnot EM, Djuric D, et al. Soluplus as an effective absorption enhancer of poorly soluble drugs in vitro and in vivo. Eur J Pharm Sci 2012;45(03):336-343

33 Lian X, Dong J, Zhang J, et al. Soluplus based 9-nitrocamptothecin solid dispersion for peroral administration: preparation, characterization, in vitro and in vivo evaluation. Int J Pharm 2014;477 $(1,2): 399-407$

34 Guo Y, LuoJ, Tan S, Otieno BO, Zhang Z. The applications of vitamin E TPGS in drug delivery. Eur J Pharm Sci 2013;49(02):175-186 